\title{
Reliability of Calf Bioelectrical Impedance Spectroscopy and Magnetic-Resonance-Imaging-Acquired Skeletal Muscle Hydration Measures in Healthy People
}

\author{
Anuradha Sawant, ${ }^{1,2}$ Andrew A. House, ${ }^{3}$ Bert M. Chesworth, ${ }^{4}$ Joseph Gati, ${ }^{5}$ \\ Robert Lindsay, ${ }^{3}$ Denise M. Connelly, ${ }^{6}$ Robert Bartha, ${ }^{5}$ and Tom J. Overend ${ }^{6}$ \\ ${ }^{1}$ Graduate Program in Health and Rehabilitation Sciences, Physical Therapy Field, Western University, London, ON, Canada N6G $1 H 1$ \\ ${ }^{2}$ London Health Sciences Center, University Hospital Campus, 339 Windermere Road, London, ON, Canada N6A 5A5 \\ ${ }^{3}$ Western University Division of Nephrology, London Health Sciences Centre, 339 Windermere Road, London, ON, Canada N6A 5A5 \\ ${ }^{4}$ School of Physical Therapy and Department of Epidemiology and Biostatistics, Western University, London, ON, Canada N6G $1 H 1$ \\ ${ }^{5}$ The Centre for Functional and Metabolic Mapping (CFMM), Robarts Research Institute, Western University, 100 Perth Drive, \\ London, ON, Canada N6A 5 K8 \\ ${ }^{6}$ School of Physical Therapy, Western University, London, ON, Canada N6G $1 \mathrm{H1}$
}

Correspondence should be addressed to Anuradha Sawant; anuradha.sawant@lhsc.on.ca

Received 3 April 2013; Revised 17 June 2013; Accepted 17 June 2013

Academic Editor: Gary Lopaschuk

Copyright (C) 2013 Anuradha Sawant et al. This is an open access article distributed under the Creative Commons Attribution License, which permits unrestricted use, distribution, and reproduction in any medium, provided the original work is properly cited.

\begin{abstract}
Purpose. The purpose of this study was to investigate the test-retest reliability, relative variability, and agreement between calf bioelectrical impedance-spectroscopy (cBIS) acquired extracellular fluid (ECF), intracellular fluid (ICF), total water and the ratio of ECF : ICF, magnetic-resonance-imaging (MRI) acquired transverse relaxation times $\left(T_{2}\right)$, and apparent diffusion coefficient (ADC) of calf muscles of the same segment in healthy individuals. Methods. Muscle hydration measures were collected in 32 healthy individuals on two occasions and analyzed by a single rater. On both occasions, MRI measures were collected from tibialis anterior (TA), medial (MG), and lateral gastrocnemius (LG) and soleus muscles following the cBIS data acquired using XiTRON Hydra 4200 BIS device. The intraclass correlation coefficients $\left(\mathrm{ICC}_{2,1}\right)$, coefficient of variation $(\mathrm{CV})$, and agreement between MRI and cBIS data were also calculated. Results. $\mathrm{ICC}_{2,1}$ values for $\mathrm{cBIS}, T_{2}$, and ADC ranged from 0.56 to $0.92,0.96$ to 0.99 , and 0.05 to 0.56 , respectively. Relative variability between measures (CV) ranged from 14.6 to $25.6 \%$ for the cBIS data and 4.2 to $10.0 \%$ for the MRIacquired data. The ratio of ECF : ICF could significantly predict $T_{2}$ of TA and soleus muscles. Conclusion. MRI-acquired measures of $T_{2}$ had the highest test-retest reliability of muscle hydration with the least error and variation on repeated testing. Hence, $T_{2}$ of a muscle is the most reliable and stable outcome measure for evaluating individual muscle hydration.
\end{abstract}

\section{Introduction}

Chronic kidney disease is a worldwide public health problem with adverse outcomes of kidney failure and cardiovascular disease [1]. In its end stages, management of kidney disease requires renal replacement therapies such as hemodialysis (HD), peritoneal dialysis, or kidney transplant.

Hemodialysis is considered to have more pronounced deleterious effects on skeletal muscle than peritoneal dialysis [2]. The consequences of HD on physical function and quality of life have been well documented [3]. In order to offset such effects, exercise interventions have been strongly recommended to improve muscle function and reduce the risk of falls [4]. Recent systematic reviews and meta-analyses of exercise training in participants diagnosed with end-stage renal disease (ESRD) undergoing HD support the use of resisted and/or aerobic exercise to promote limb muscle strengthening [5]. These benefits were small and inconsistent with only one in nine persons receiving the benefits of exercise interventions [6]. 
Extra body water accruing during the interdialytic periods has been reported to accumulate mainly in the extracellular spaces $[7,8]$. Hemodialysis or ultrafiltration is aimed at achieving normal state of hydration [9]. However, factors such as weight gain associated with improved appetite and nutritional support [10], may influence estimation of true "dry weight" or what weight the person would be when all the extra fluid is removed from the body [11]. This may lead to dehydration or relative volume depletion [12]. Clinical symptoms such as hypotension and intradialytic cramps have been associated with volume depletion or dehydration [13]. Such HD related dehydration or volume depletion has not yet been linked to poor response to exercise interventions or fatigue in this population.Using single-frequency bioelectrical impedance analysis, Jain and Lindsay [14] have demonstrated loss of leg and arm extracellular fluid (ECF) in participants receiving conventional $\mathrm{HD}$ (three times a week) that returned to baseline at 48 hours. They suggested that the majority of fluid loss and refilling takes place in the extremities to maintain the central blood volume. Consequently, change in pre- and post-HD body mass cannot be used to establish hydration of a limb or single muscle. Hence, assessing hydration of the specific muscle may provide insight and understanding of the role of HD-related fluctuating hydration on skeletal muscle function in people with $\mathrm{ESRD} / \mathrm{HD}$; as in athletes, dehydration of $1 \%$ to $2 \%$ of total body weight begins to compromise an array of physiologic functions and negatively influences performance and force generation [15].

Several methods have been used to estimate hydration of the human body. Bioelectrical impedance spectroscopy (BIS) is a safe and inexpensive in vivo method for whole body or segmental composition assessment providing estimates of ECF and intracellular (ICF) fluid volume shifts [16]. While BIS can provide estimates of ECF and ICF for the whole body or a segment of the body, it does not provide such estimates for a specific muscle, which limits its utility to ascertain the adequacy of $\mathrm{HD}$ at a muscle level.

Magnetic resonance imaging (MRI) acquired images provide quantitative measures of skeletal muscle fibre type composition and transverse relaxation time constants $\left(T_{2}\right)$ [17]. Researchers have used $T_{2}\left(T_{2}\right.$ : a measure of transverse magnetization signal decay) to investigate the changes in ECF volume or extracellular space ratio following drug-induced myopathy [18], neural damage [19, 20], and exercise [21]. Magnetic resonance imaging also provides an apparent diffusion coefficient (ADC), measured by a diffusion-weighted imaging technique, which is sensitive to the hydrodynamic environment of the tissue fluid volume [22].

Test-retest reliability of BIS has wide variability in the reliability coefficient for TW, ECF, and ICF [23]. Establishing such test-retest reliability of cBIS measures and $T_{2}$ and $\mathrm{ADC}$ for accurate estimates of true differences in skeletal muscle hydration in healthy people with varying levels of physical activity, age, and body composition is essential to determine the most reliable measure of muscle hydration that can be applied in the population with ESRD/HD [24]. Thus the primary purpose of this study was to evaluate the psychometric properties of cBIS measures and MRI-acquired
$T_{2}$ and ADC. The secondary objectives were to (1) compare the relative variations of the cBIS and MRI-acquired measures of hydration and (2) evaluate agreement between these two methods of acquiring estimates of muscle hydration. Based on prior-published reports on relationship between the ECF and $T_{2}$, we evaluated agreement between the ratio of ECF : ICF and $T_{2}$ of the tibialis anterior (TA), lateral (LG), and medial (MG) gastrocnemius and soleus muscles within the same calf segment.

\section{Methods}

2.1. Sample Size. A required sample of 32 participants was calculated as suggested by Donner and Eliasziw [25], based on alpha $=0.05$, statistical power of $80 \%$, and a correlation of greater than 0.9 for a single observer on two occasions.

2.2. Inclusion Criteria. Eligibility to participate in the study was determined as follows.

(1) Participants were healthy adults 18 years of age or older.

(2) They were able to understand instructions in English and provide informed consent.

(3) They had no health condition, existence of a foreign body or medical device that would preclude them from having an MRI.

Participants not meeting all of the inclusion criteria were excluded from the study. Western University's Ethics Review Board for Human Subjects granted ethics approval for this study and all participants provided written informed consent prior to participation.

2.3. Questionnaires. Levels of self-reported physical activity were quantified using the Human Activity Profile Questionnaire (HAP) [26] administered to participants using a question and answer or interview format. A maximum activity score (MAS-HAP) (the highest-numbered activity the person reports still doing) and an adjusted activity score (AASHAP) calculated by subtracting the number of activities marked as "stopped doing this activity" listed below the MAS-HAP were generated using the HAP questionnaire. The HAP consisted of 94 activities, ranked in ascending order of difficulty according to the energy requirements of the task. The presence of any comorbidity was determined using the Charlson comorbidity index [27] calculated using the automated calculator.

2.4. Skeletal Muscles. Tibialis anterior, a foot dorsiflexor muscle, was chosen to represent a muscle from the anterior compartment of the lower leg, and MG, LG, and soleus were selected to represent muscles from the posterior compartment [28].

2.5. Calf Bioelectrical Impedance Spectroscopy. A multifrequency device (XiTRON 4200, Xitron Technologies, San Diego, CA, USA) was used for automatic continuous sequential measurements of calf with frequencies ranging from 


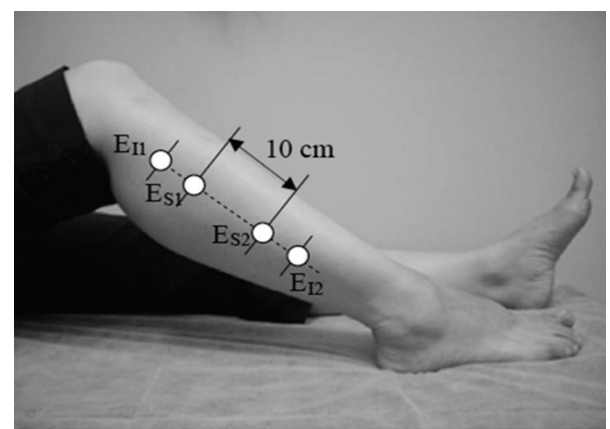

FIGURE 1: Electrode placement for measurement of calf-multifrequency bioelectrical impedance spectroscopy. $\mathrm{E}_{\mathrm{S} 1}$ : first measuring electrode placed at calf-maximum circumference. $\mathrm{E}_{\mathrm{S} 2}$ : second measuring electrode $10 \mathrm{cms}$ from the first electrode. $\mathrm{E}_{\mathrm{I} 1}$ : injection electrode $1 . \mathrm{E}_{\mathrm{I} 2}$ : injection electrode 2 .

$5 \mathrm{kHz}$ to $1 \mathrm{MHz}$. Measures of ECF, ICF, TW, and ECF : ICF ratios for the calf were calculated as estimates of hydration. Two measuring $\left(\mathrm{E}_{\mathrm{S} 1}\right.$ and $\left.\mathrm{E}_{\mathrm{S} 2}\right)$ and two injecting electrodes $\left(\mathrm{E}_{\mathrm{I} 1}\right.$ and $\left.\mathrm{E}_{\mathrm{I} 2}\right)$ were placed on the lateral side of the tested leg. The $\mathrm{E}_{\mathrm{S} 1}$ electrode was placed at maximum circumference of the calf; $\mathrm{E}_{\mathrm{S} 2}$ was placed $10 \mathrm{cms}$ distal to $\mathrm{E}_{\mathrm{S} 1}$. Injecting electrode $\mathrm{E}_{\mathrm{I} 1}$ was placed $5 \mathrm{cms}$ proximal to $\mathrm{E}_{\mathrm{S} 1}$ and $\mathrm{E}_{\mathrm{I} 2}$ was placed $5 \mathrm{cms}$ distal to $\mathrm{E}_{\mathrm{S} 2}$ (Figure 1). A fiduciary marker (vitamin $\mathrm{E}$ capsule) was placed at the $\mathrm{E}_{\mathrm{S} 1}$ electrode site for the identification of the first measuring electrode on MRI. Each measurement was repeated at least 10 times and the average value was used in subsequent computation of the calf hydration estimate for that test occasion. Calculations and curve fitting (Cole-Cole model) were done offline as described by Zhu et al. [29].

2.6. Magnetic Resonance Imaging. All MRI-acquired data were collected on a 3.0 Tesla Tim Trio whole body imaging system (Siemens, Erlangen, Germany) using an 8-channel knee coil. A multiecho spin-echo (8 echoes) volume (11 contiguous $3 \mathrm{~mm}$ transverse slices; $160 \mathrm{~mm}$ field of view; $384 \times 384$ matrix; TE $(13.1 \mathrm{~ms}$ to $93.6 \mathrm{~ms})$; TR $=1500 \mathrm{~ms})$ and a diffusion-weighted volume $\left(b=400 \mathrm{~mm} / \mathrm{s}^{2} ; 6\right.$ directions; 22 contiguous $4 \mathrm{~mm}$ transverse slices; $160 \mathrm{~mm}$ field of view; $128 \times 128$ matrix; TE $=61.6 \mathrm{~ms}$; TR $=6200 \mathrm{~ms} ; 8$ averages) were used to measure the $T_{2}$ and ADC, respectively, of the TA, MG, LG, and soleus muscles.

The $T_{2}$ maps for the muscles of interest were generated in OsiriX using the " $T_{2}$ Fit Map" plugin by importing their crosssectional areas outlined (closest to the fiduciary marker) on the $T_{2}$-weighted images. On this map, three areas or regions of interest (ROI) less than $0.22 \mathrm{~cm}^{2}$ were selected taking care to avoid visible subcutaneous fat, septum, or neurovascular bundles [30] (Figure 2). We sampled three areas of a muscle and values were averaged to obtain a representative $T_{2}$ for each muscle.

Calculations of ADC for each of the four muscles were completed using the CSA of muscle as outlined in the $T_{2}$ weighted image and imported into the ADC map generated using OsiriX plugin "ADC Map" (Figure 3) [22]; the software automatically calculated and displayed the ADC values.

2.7. Data Collection. Data were collected on two different occasions at least one day apart preferably at the same time of day. Participants were asked to refrain from exercise and alcohol for 8 to 10 hours and not eat or drink anything for two hours prior to testing to control extraneous factors affecting hydration of the muscle/body for short durations [23]. Participants were positioned in supine lying on a standard hospital bed for 30 minutes prior to imaging to allow redistribution of water to the lower extremities [31]. Measurements of hydration using cBIS were collected while the participants were lying supine in preparation for MRI. All MRI and cBIS-acquired measures of hydration were collected from the same calf segment.

\subsection{Data Analysis}

2.8.1. Statistical Analysis. Participant demographic data, including age, average body mass, body mass index, Human Activity Profile scores [26], and Charlson Comorbidity Index [27] were analyzed using descriptive statistics and reported as group data with means and standard deviations. The testretest reliability for all measures of hydration (ECF, ICF, TW, ECF:ICF, $T_{2}$, and ADC measures for TA, LG, MG, and soleus were assessed using two-way random effects intraclass correlation coefficients $\left(\mathrm{ICC}_{2,1}\right)$ [32]. An $\mathrm{ICC}_{2,1} \geq$ 0.9 was considered "excellent," and values $\geq 0.8$ and $<0.9$ were considered "good" and those below 0.8 were considered to be of questionable clinical significance [33]. Minimal detectable change values at $95 \%$ confidence $\left(\mathrm{MDC}_{95}\right)$ were calculated using the formula: $\mathrm{MDC}_{95}=\mathrm{SEM} \times \sqrt{ } 2 \times 1.96$, where SEM is the standard error of measurement calculated from the $\mathrm{ICC}_{2,1}$ analysis of variance tables [34]. The $\mathrm{MDC}_{95}$ is an estimate of the amount by which $95 \%$ of participants who remain unchanged will display random fluctuations. The variability in their change scores is expected to be within the bounds of $\mathrm{MDC}_{95}$ value [34]. The SEM estimates how repeated measures of a participant tend to be distributed around the true score [35]. For each measure, the difference between the measurements obtained on two occasions was plotted against the mean of those two measurements. The 95\% limits of agreement (LOA) were estimated by the mean difference between the two occasions, $\bar{d}$, and the standard deviation of that difference $(s)$. Assuming a Gaussian distribution, 95\% of the differences were calculated to lie between $\bar{d}-1.96 \mathrm{~s}$ and $\bar{d}+1.96 s$ [24]. Coefficient of variation (CV) for each measure was computed using statistical software. Levene's test, used to evaluate the homogeneity of variances between the cBIS and MRI-acquired measures, was computed by using one-way analysis of variance on the absolute deviation of each score from the mean of its group [36]. The agreement between ECF: ICF and MRI-acquired $T_{2}$ and ADC of TA, LG, MG, and soleus was evaluated using linear regression analysis [37]. A statistical software package (IBM SPSS v20.0) was used for all data analyses and Prism 4.0a for Macintosh (GraphPad Software Inc.) was used for plotting and analyses 

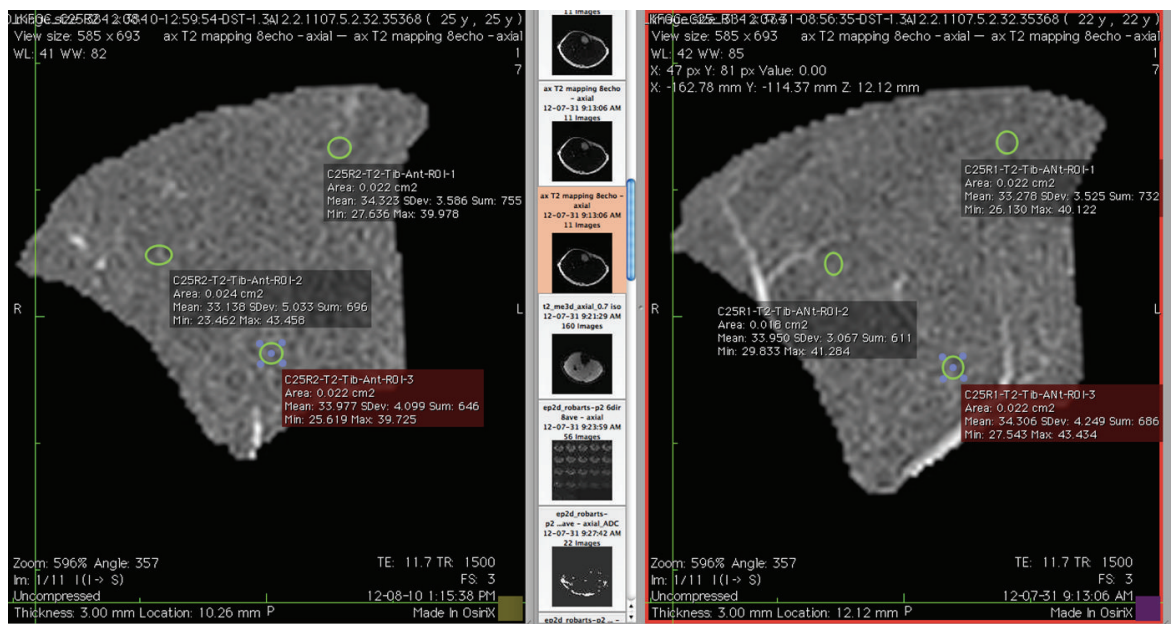

FIgURE 2: Example of measuring transverse relaxation times of tibialis anterior.

Image of skeletal muscles outlined on $T_{2}$-weighted image Tibialis anterior

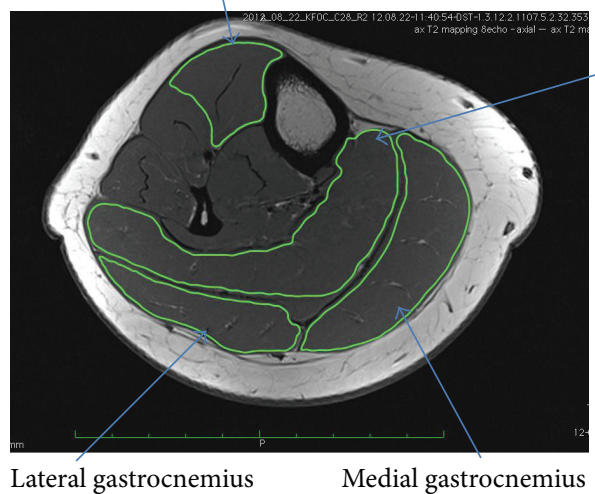

Tibialis anterior

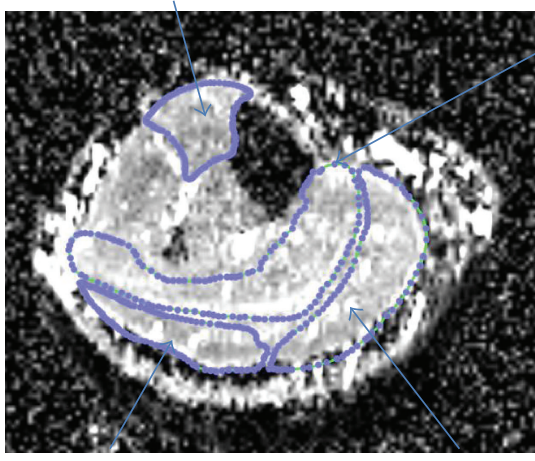

Lateral gastrocnemius Medial gastrocnemius Soleus

FIGURE 3: Example of measuring apparent diffusion coefficient (ADC).

of differences-against-mean plots. Statistical significance was assumed for $P$ values $<0.05$.

\section{Results}

3.1. Subjects. Thirty-two participants (16 men and 16 women) meeting the inclusion criteria were recruited for this study. Table 1 summarizes the characteristics of these participants.

3.2. Reliability of Measures of Muscle Hydration. Results for the measures of hydration for time one and two are presented as group means and standard deviations in Table 2. Values for the $\mathrm{ICC}_{2,1}$, the $\mathrm{MDC}_{95}$, bias, and the $95 \% \mathrm{LOA}$ are presented for each measure of hydration in Tables from 3 to 5 . For the test-retest reliability of the cBIS measures (Table 3), ECF : ICF had the lowest reliability, while ICF and TW had the highest. For $T_{2}$, the average of three ROI yielded better test-retest reliability than estimates derived from a single ROI (Table 4). $\mathrm{ICC}_{2,1}$ values for single measures of ADC of the leg muscles varied between 0.4 and 0.5 (Table 5). However, for average measures of $\mathrm{ADC}, \mathrm{ICC}_{2,1}$ ranged from 0.6 to 0.7 .
TABLE 1: Characteristics of the participants included in this study.

\begin{tabular}{lc}
\hline Participant characteristic & Group mean $(\mathrm{SD})$ \\
\hline Weight $(\mathrm{kg})$ & $75.27(13.24)$ \\
Height $(\mathrm{cms})$ & $168.1(8.6)$ \\
BMI $\left(\mathrm{kg} / \mathrm{m}^{2}\right)$ & $26.3(3.3)$ \\
Charlson comorbidity index & $0.16(0.72)$ \\
HAP-MAS & $87.8(5.3)$ \\
HAP-AAS & $85.4(8.3)$ \\
\hline
\end{tabular}

AAS: adjusted activity score; BMI: body mass index; HAP: human activity profile; SD: standard deviation.

3.3. Limits of Agreement between Repeated Measures of Hydration. The difference-against-mean plots of ICF and TW indicate a larger positive bias at the lower end of the scale than the smaller bias at higher values of ICF and TW. The plot of the ECF : ICF ratio (Figure 4) indicates increasing bias at higher values of the ratio. The plots of $T_{2}$ (Figure 5) for all the muscles do not reveal any systematic bias; however, a single data point for $T_{2}$ of TA and three data points for soleus were 
TABLE 2: Estimates of lower leg and individual skeletal muscle hydration using bioelectrical impedance spectroscopy and magnetic resonance spectroscopy in healthy adults $(n=32)$.

\begin{tabular}{lcc}
\hline Measure & $\begin{array}{c}\text { Time 1 } \\
\text { mean (SD) }\end{array}$ & $\begin{array}{c}\text { Time 2 } \\
\text { mean }(S D)\end{array}$ \\
\hline BIS (Liters) & $0.15(0.02)$ & $0.15(0.02)$ \\
ECF & $0.49(0.15)$ & $0.48(0.15)$ \\
ICF & $0.64(0.16)$ & $0.62(0.17)$ \\
TW & $0.31(0.09)$ & $0.31(0.06)$ \\
ECF $:$ ICF & & \\
$T_{2}$ (ms) & $36.37(1.42)$ & $36.25(1.58)$ \\
Tibialis anterior & $38.73(3.31)$ & $38.79(3.34)$ \\
Med gastrocnemius & $40.19(3.2)$ & $40.31(2.96)$ \\
Lat gastrocnemius & $39.68(2.45)$ & $39.81(2.37)$ \\
Soleus & & \\
ADC (mm/s $\left.{ }^{2}\right)$ & $1.51(0.11)$ & $1.50(0.08)$ \\
Tibialis anterior & $1.59(0.09)$ & $1.59(0.06)$ \\
Med gastrocnemius & $1.64(0.10)$ & $1.63(0.06)$ \\
Lat gastrocnemius & $1.55(0.1)$ & $1.54(0.1)$ \\
Soleus &
\end{tabular}

ADC: apparent diffusion coefficient; BIS: bioelectrical impedance spectroscopy; ECF: extracellular fluid; ICF: intracellular fluid; TW: total water; Med: medial; Lat: lateral.

observed to lie outside the 95\% LOA. For the ADC, the plots (Figure 6) for all the muscles did not identify any systematic bias.

3.4. Comparison of Variances in cBIS and MRI-Acquired Measures of Hydration. The coefficient of variation for each measure on occasions one and two is presented in Table 6. The Levene's test for equality of variances between the measures was significant $\left(F_{23,744}=18.405 ; P<0.001\right)$. Post hoc comparisons using Tukey's HSD indicated that the differences in mean variation between the MRI acquired $T_{2}$ and $A D C$ of the muscles investigated and ICF, TW and ratio of ECF : ICF were significant. However, these differences were not significant for $T_{2}$ of $\mathrm{LG}, \mathrm{MG}$, and soleus, $\mathrm{ADC}$ of all the muscles, and ECF.

3.5. Agreement between cBIS- and MRI-Acquired Measures of Hydration. Ratio of ECF : ICF was significantly associated with $T_{2}$ of TA ( $\beta=0.4, P=0.041 ; b=32.9$ (95\% CI: 31.2, $\left.36.1), t_{(30)}=20.38 P<0.05\right)$ and soleus $(\beta=0.4, P=0.018$; $b=33.8$ (95\% CI: 28.8, 36.1), $t_{(30)}=13.75, P<0.05$ ); ratio of ECF : ICF explained a significant proportion of $T_{2}$ of TA $\left(R^{2}=0.13, F(1,30)=4.56, P<0.05\right)$ and soleus $\left(R^{2}=0.17\right.$, $F(1,30)=6.24, P<0.05)$ (Figure 7). The agreement between ECF : ICF and $T_{2}$ of LG and MG and ADC of all the muscles investigated was nonsignificant (Table 7).

\section{Discussion}

To our knowledge this is the first study that has established test-retest reliability of the cBIS- and MRI-acquired measures of skeletal muscle hydration, namely, $T_{2}$ and ADC. The results of this study indicate that $T_{2}$ time constants estimating hydration of muscles of the lower leg have excellent reliability and reproducibility when compared to cBIS and ADC. This study also established $\mathrm{MDC}_{95}$ values indicating random fluctuations in the measures of hydration in $95 \%$ of unchanged participants on repeated measurements. According to our results, an $\mathrm{MDC}_{95}$ of $0.96 \mathrm{~ms}$ between repeated measures of $T_{2}$ of LG indicates a minimum fluctuation of the measure one should expect to see in $95 \%$ of the participants whose measurements randomly fluctuate in repeated measurements.

Based on the characteristics of the participants recruited in this study, the results of this study can be generalized to a wide range of healthy and active adults. The age of the participants in this study ranged from 22 to 81 years and the activity profile or the HAP scores ranged from 75 to 94 . The activity levels of the population can impact the BIS measures [38], and hence we recruited active participants to reduce variability in cBIS measures.

Our findings of good-to-excellent reliability of the cBIS measures are in accordance with those reported in the literature for the repeated measurements of whole body and segmental BIS [23]. Several factors can impact the testretest reliability of cBIS-acquired data. These include dayto-day variation in body hydration [23], exercise [21,39], participants' body type, and equations utilized to estimate ECF, ICF, and TW. The reliability of the ECF:ICF may have been affected by the differences in body types of our participants (BMI $25.9 \pm 2.8$ ), and differences in offline model fitting (range from 0 to 2 ) affecting ECF volume calculations of these participants. These findings are supported by a difference-against-the-mean-plot which suggests that the errors in estimating ECF: ICF were influenced by the value of the ratio; that is, the errors in measurements were larger at higher values of ECF:ICF ratio and vice versa. Hence contribution of the differences between measurements may not be associated with technical differences or operator error. Kyle et al. [23] have reported similar findings in studies evaluating whole body composition.

Our findings of excellent $\mathrm{ICC}_{2,1}$ for $T_{2}$ time constants of all the muscles investigated indicate the ability of the measure to differentiate between the study participants. Factors such as exercise [21] and perhaps variation in placement of the limb in the MRI receiver coil influencing signal-to-noise ratio may impact MRI-acquired measures as well. In this study, about $4 \%$ of the measurements for $T_{2}$ of soleus lay outside the $95 \%$ limits of agreement; one was lower and two higher than the limits of agreement. Two of these participants had performed light physical activity just prior to their scheduled data collection session, and perhaps 30 minutes of supine positioning may not have been adequate to equilibrate the muscle ECF and ICF associated with effects of physical activity and led to the differences in the $T_{2}$ of soleus between the two occasions [39]. The increments in $T_{2}$ of the soleus on repeated measurements can be supported by findings of Nygren and Kaijser [21] in participants who performed light exercises using a 1.5 Tesla magnet and four echoes. For the difference in repeated measures of $T_{2}$ of gastrocnemius muscle for the nonexercising leg they observed a change of 
TABLE 3: Test-retest reliability of estimates of calf muscle hydration in healthy adults $(n=32)$ using bioelectrical impedance spectroscopy values.

\begin{tabular}{|c|c|c|c|c|}
\hline Estimate of calf muscle hydration & $\mathrm{ICC}_{2,1}(95 \% \mathrm{CI})$ & $\mathrm{MDC}_{95}$ (Liters) & Bias (SD) & $95 \%$ LOA \\
\hline ECF & $0.77(0.58,0.88)^{*}$ & - & $-0.004(0.14)$ & $-0.03,0.02$ \\
\hline ICF & $0.90(0.8,0.95)^{*}$ & 0.19 & $0.02(0.05)$ & $-0.08,0.12$ \\
\hline TW & $0.92(0.84,0.96)^{*}$ & 0.19 & $0.02(0.06)$ & $-0.09,0.12$ \\
\hline ECF : ICF & $0.58(0.29,0.77)^{*}$ & 0.15 & $-0.01(0.07)$ & $-0.15,0.12$ \\
\hline
\end{tabular}

${ }^{*} P<0.001$.

ECF: extracellular fluid; ICC: intraclass correlations; ICF: intracellular fluid; LOA: limits of agreement; MDC: minimal detectable change; SD: standard deviation; TW: total water.

TABLE 4: Test-retest reliability of transverse relaxation times.

\begin{tabular}{|c|c|c|c|c|c|c|}
\hline Muscle & Single measure $(95 \% \mathrm{CI})$ & $\begin{array}{c}\text { Average } \\
(2 \text { measures }) \\
(95 \% \mathrm{CI})\end{array}$ & $\begin{array}{c}\text { Average } \\
(3 \text { measures }) \\
(95 \% \mathrm{CI})\end{array}$ & $\mathrm{MDC}_{95}(\mathrm{~ms})$ & Bias (SD) & $95 \% \mathrm{LOA}$ \\
\hline Tibialis anterior & $0.87(0.76,0.94)^{*}$ & $\begin{array}{c}0.93(0.86 \\
0.96)^{*}\end{array}$ & $0.93(0.87,0.97)^{*}$ & 1.06 & $0.09(0.56)$ & $-0.99,1.18$ \\
\hline Lat gastrocnemius & $0.98(0.95,0.98)^{*}$ & $\begin{array}{c}0.97(0.95 \\
0.99)^{*}\end{array}$ & $\begin{array}{c}0.99(0.97 \\
0.99)^{*}\end{array}$ & 1.29 & $-0.12(0.55)$ & $-1.19,0.96$ \\
\hline Med gastrocnemius & $0.95(0.9,0.98)^{*}$ & $\begin{array}{c}0.97(0.94 \\
0.99)^{*}\end{array}$ & $\begin{array}{c}0.99(0.97 \\
0.99)^{*}\end{array}$ & 0.94 & $-0.05(0.29)$ & $-0.62,0.52$ \\
\hline Soleus & $0.89(0.78,0.94)^{*}$ & $\begin{array}{c}0.94(0.88 \\
0.97)^{*}\end{array}$ & $\begin{array}{c}0.96(0.92 \\
0.98)^{*}\end{array}$ & 0.29 & $-0.03(0.96)$ & $-1.85,1.90$ \\
\hline
\end{tabular}

${ }^{*} P<0.001$

ICC: intraclass correlation; Lat: lateral; LOA: limits of agreement; MDC: minimal detectable change; Med: medial; SD: standard deviation.

TABLE 5: Test-retest reliability of apparent diffusion coefficients.

\begin{tabular}{|c|c|c|c|c|c|}
\hline Muscle & ICC $_{2,1}(95 \% \mathrm{CI})$ single measure & $\mathrm{ICC}_{2,1}(95 \% \mathrm{CI})$ average measures & $\mathrm{MDC}_{95}\left(\mathrm{~mm} / \mathrm{s}^{2}\right)$ & Bias (SD) & 95\% LOA \\
\hline Tibialis anterior & $0.40(0.06,0.67)^{*}$ & $0.57(0.13,0.79)^{*}$ & 0.96 & $0.03(0.10)$ & $-0.17,0.23$ \\
\hline Lateral gastrocnemius & $0.46(0.13,0.69)^{*}$ & $0.63(0.23,0.82)^{*}$ & 0.88 & $0.132(0.74)$ & $-1.31,1.57$ \\
\hline Medial gastrocnemius & $0.45(0.12,0.69)^{*}$ & $0.56(0.22,0.82)^{*}$ & 0.09 & $0.008(0.078)$ & $-0.15,0.16$ \\
\hline Soleus & $0.51(0.19,0.73)^{*}$ & $0.67(0.32,0.84)^{*}$ & 0.13 & $0.01(0.11)$ & $-0.19,0.22$ \\
\hline
\end{tabular}

${ }^{*} P<0.05$.

CI: confidence interval; ICC: intraclass correlation; LOA: limits of agreement; MDC: minimal detectable change; SD: standard deviation.

TABLE 6: Coefficient of variability of cBIS and MRI-acquired measures of hydration at time one and two.

\begin{tabular}{|c|c|c|c|c|}
\hline & $T_{2}-1$ & $T_{2}-2$ & ADC-1 & ADC-2 \\
\hline Tibialis anterior & 4.2 & 4.5 & 6.5 & 6.1 \\
\hline Lateral gastrocnemius & 9.0 & 8.4 & 7.2 & 7.6 \\
\hline Medial gastrocnemius & 10.0 & 10.0 & 5.4 & 5.2 \\
\hline Soleus & 8.1 & 8.2 & 6.5 & 7.2 \\
\hline & \multicolumn{2}{|c|}{$\mathrm{T} 1$} & \multicolumn{2}{|c|}{$\mathrm{T} 2$} \\
\hline \multicolumn{5}{|l|}{ cBIS } \\
\hline ECF & \multicolumn{2}{|c|}{14.6} & \multicolumn{2}{|c|}{15.3} \\
\hline ICF & \multicolumn{2}{|c|}{24.9} & \multicolumn{2}{|c|}{26.0} \\
\hline TW & \multicolumn{2}{|c|}{20.7} & \multicolumn{2}{|c|}{22.6} \\
\hline Ratio (ECF : ICF) & \multicolumn{2}{|c|}{24.9} & \multicolumn{2}{|c|}{25.6} \\
\hline
\end{tabular}

ADC: apparent diffusion coefficient; cBIS: calf bioelectrical impedance spectroscopy; ECF: extracellular fluid; ICF: intracellular fluid; T1: occasion 1; T2: occasion $2 ; T_{2}$ : transverse relaxation times; TW: total water.

$0.4 \%$ to $1.0 \%$. This change is slightly higher than the change we observed on repeated measurements of $T_{2}$ of the MG and LG $(0.01 \%-0.22 \%$ calculated based on bias of 0.004 and mean of $38.73 \mathrm{~ms}$ for MG, bias of 0.09 , and mean of $40.19 \mathrm{~ms}$ for LG). These differences between our results and those reported by Nygren and Kaijser [21] could be due to several factors such as differences in the mean age of the participants (mean age of participants in the study by Nygren and Kaijser [21] was 25 yrs), number of echoes utilized for measuring $T_{2}$ (Nygren and Kaijser [21] computed $T_{2}$ by fitting four echoes), sample size (Nygren and Kaijser [21] recruited six participants), and perhaps in variation in intramuscular fat.

Our overall results indicate a smaller variation for the MRI-acquired muscle hydration measures, ranging from 4.2 to $10 \%$ whereas the same for the cBIS acquired measures ranged from 14.6 to $25.6 \%$. The variation in anatomical configurations of the muscles of the calf by age of the participants recruited in this study support our findings of minimum variability of $T_{2}$ for TA and largest variability in the ICF. Using segmental multifrequency BIS Yamada et al. [40] suggested that the expansion of ECF relative to ICF and the lean volume of the lower extremity masked actual muscle cell atrophy with aging. This supports our observations of larger relative within-subject variation for the cBIS-acquired 


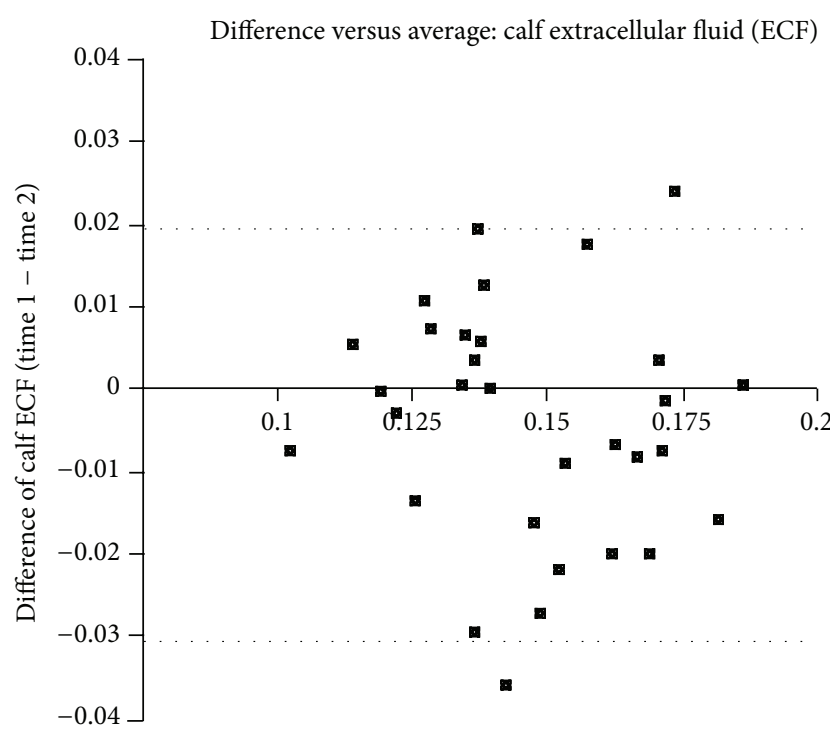

Average calf ECF (time $1+$ time 2)/2

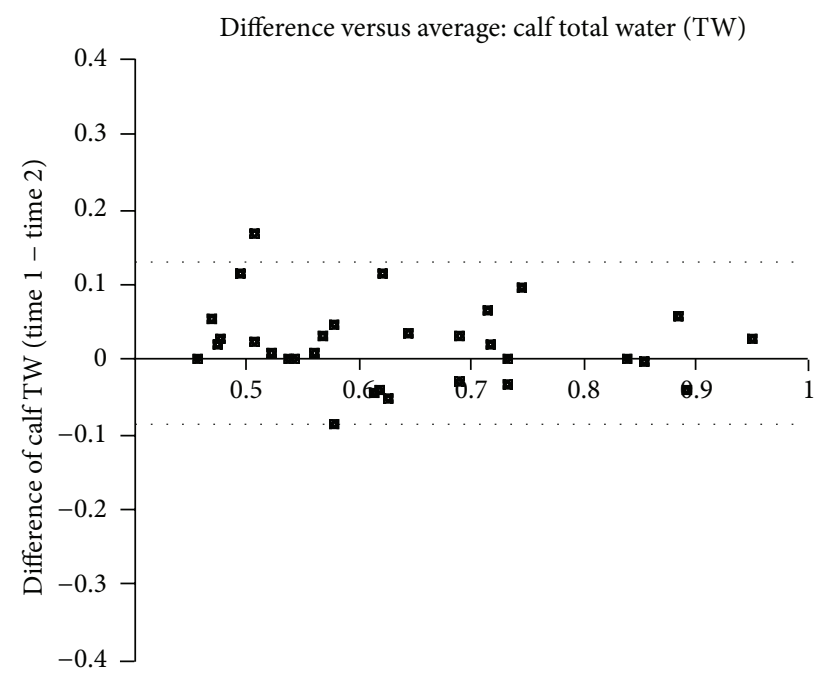

Average calf TW (time $1+$ time 2$) / 2$

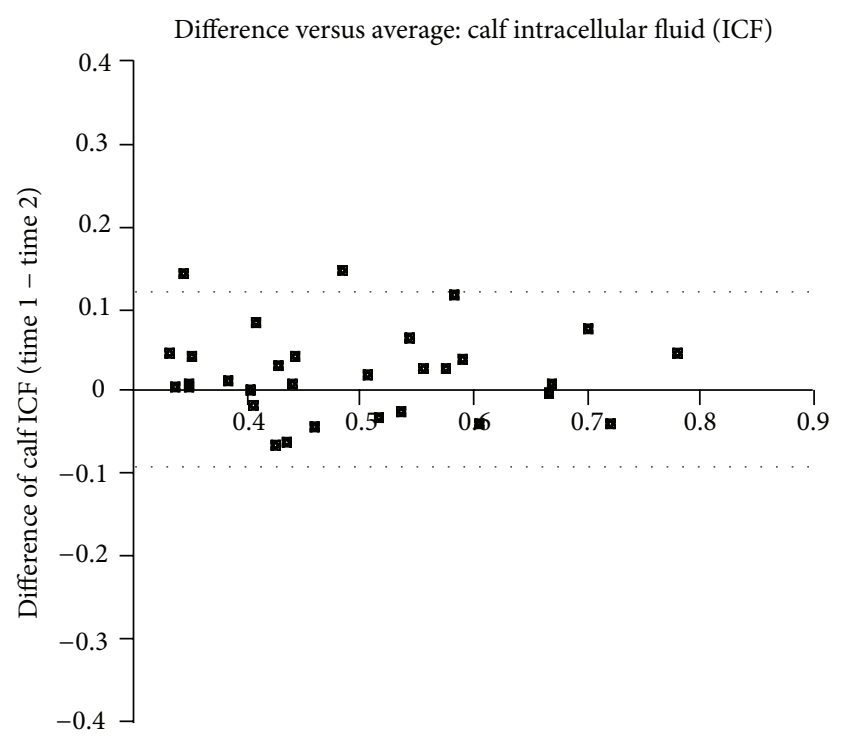

Average calf ICF (time $1+$ time 2)/2

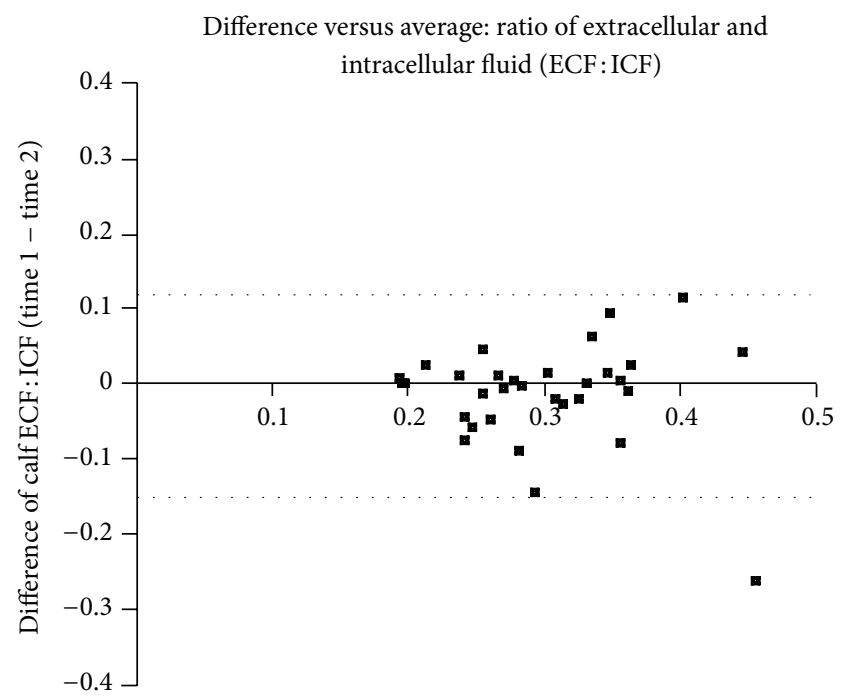

Average calf ECF : ICF (time $1+$ time 2)/2

FIGURE 4: Plots of difference versus average: calf bioelectrical impedance measures (cBIS).

measures as we recruited participants aged from 22 to 81 years.

Transverse relaxation times of muscles investigated measured changes unique to each muscle; hence numerous studies on the dorsiflexors [41-43] reporting lack of significant difference in strength and contractile properties/kinetics between the young and old support our findings of smaller within-subject variability for TA. However, according to Vandervoort and McComas [44] age-related changes in muscle strength and size were larger in the plantar flexor muscles. Hence the CV for $T_{2}$ of plantar flexor muscles (LG, MG, and soleus) was larger than that for the $T_{2}$ values of TA values for these muscles.

Joseph et al. [45] quantified reproducibility of $T_{2}$ of cartilage using coefficient of variation $(\mathrm{CV})$ which ranged from 0.83 to 3.21 . This is slightly higher than the results with $\mathrm{CV}$ for $T_{2}$ of TA (0.04) and MG (0.09). These differences could be due to variation of the morphological characteristics and hydration of knee cartilage and skeletal muscle.

Skeletal muscle is a heterogeneous structure in its composition and architecture. Measurement of hydration in a limb segment such as the calf using BIS can be considered to be at a "whole body" level, whereas MRI estimates hydration at the "tissue system level" [46]. A direct comparison of these two techniques, based on different assumptions and methods, is challenging. Besides no study has yet determined a direct relationship between $T_{2}$ and wet/dry weight of the calf/shank muscles in healthy individuals. Our results of coefficient of regressions indicate that ECF : ICF ratio explained 13 and $17 \%$ variance observed in the $T_{2}$ of TA and soleus, respectively. Several factors other than the interstitial fluid in TA may contribute to the variation in $T_{2}$ of TA and soleus. Generally 
Difference versus average: transverse relaxation times $\left(T_{2}\right)$ of

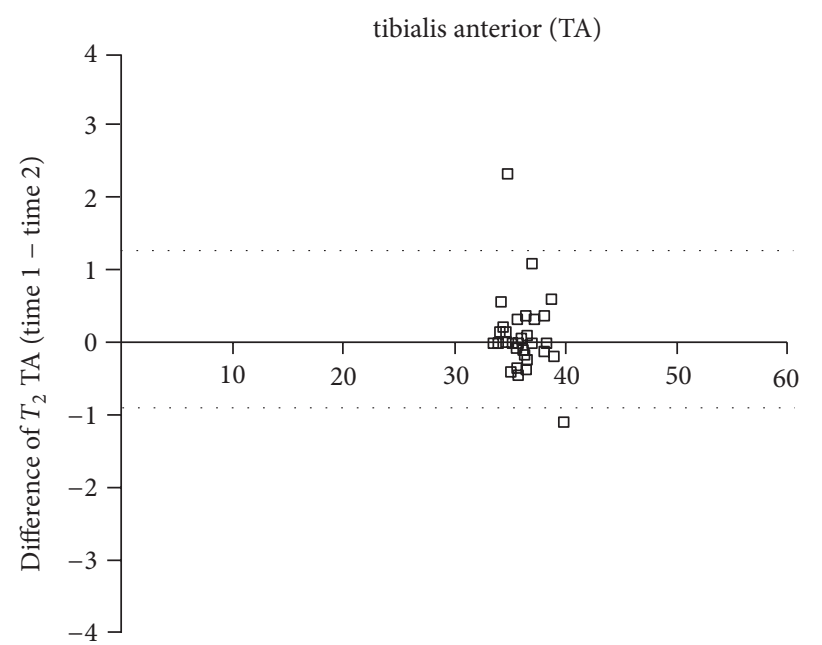

Average $T_{2}$ TA (time $1+$ time 2$) / 2$

Difference versus average: transverse relaxation times $\left(T_{2}\right)$ of

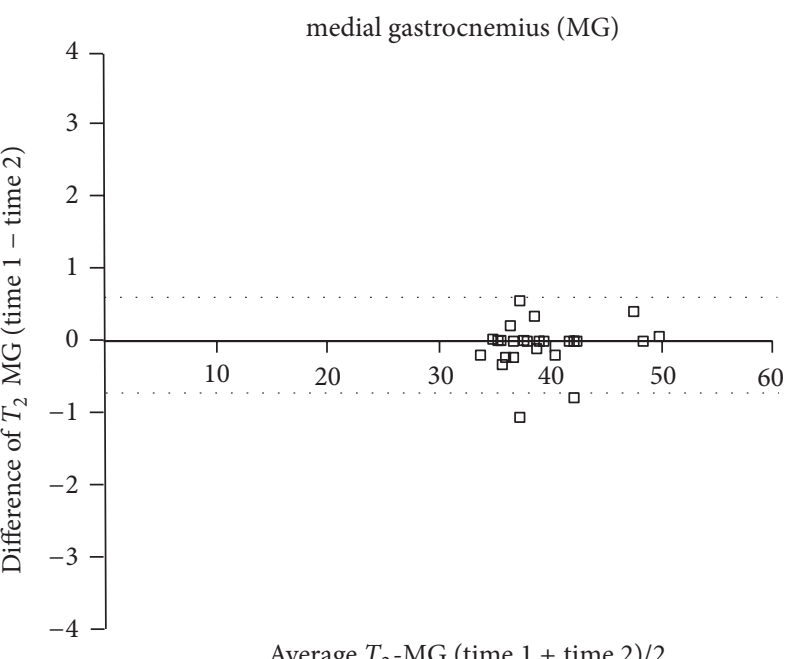

Difference versus average: transverse relaxation times $\left(T_{2}\right)$ of

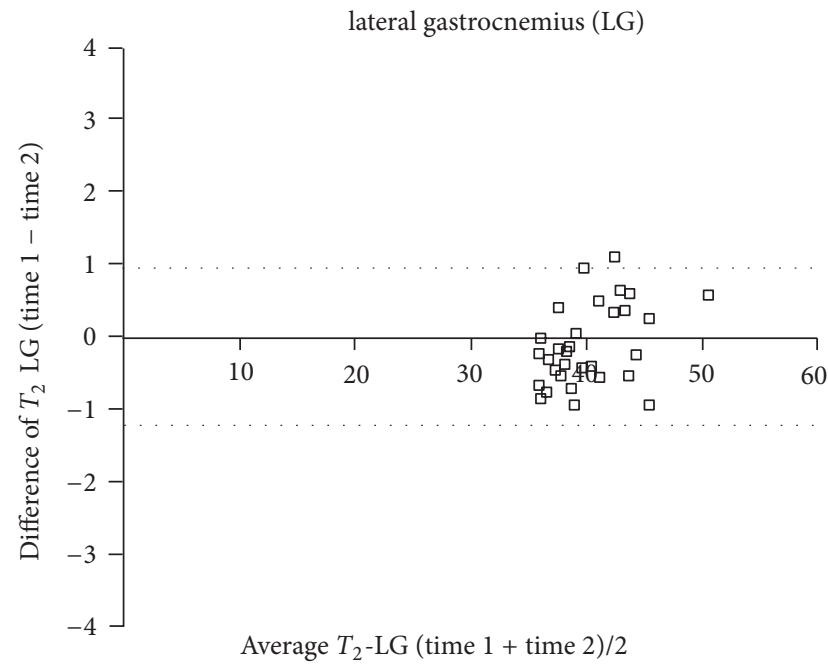

Difference versus average: transverse relaxation times $\left(T_{2}\right)$ of

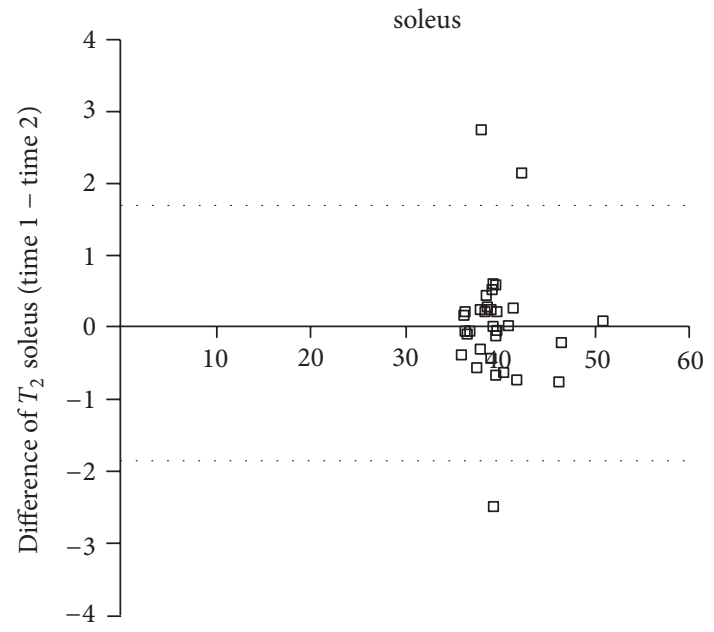

Average $T_{2}$ soleus (time $1+$ time 2$) / 2$

FIgure 5: Plots of difference versus average: $T_{2}$ relaxation times of all muscles.

$T_{2}$ values correlate most strongly with bulk water content of the tissue [47]. Tissues with high concentrations of aliphatic lipid protons have longer $T_{2}$. Hence MRI signals arising from bulk water content or total water and the degree of binding to the lipids may result in alterations of $T_{2}$. Since fats/lipids hold about $10-15 \%$ of total water [48], the impact of possible increase to intra- and extramyocellular lipids and the amount of water bound within these lipids may have impacted our goodness-of-fit between $T_{2}$ of TA and soleus and ECF : ICF ratio; $T_{2}$ accounting for the water bound to lipids and ECF : ICF excluding this portion of water. We did not correct our data prior to analysis for the changes in $T_{2}$ associated with possible increase in intra- and extramyocellular lipids in this population. However we attempted to control for these changes by choosing small ROIs for analyses.

Factors other than the interstitial fluid in TA and soleus may contribute to the estimates of ECF: ICF ratio such as plasma volume in the large vessels, namely, the popliteal artery, anterior tibial artery, saphenous veins, and intra and extracellular spaces embedded within the other muscles of the calf, namely, LG, MG, and peronei. The contribution of the interstitial space encompassed by TA and soleus to the ECF and ICF may perhaps also be related to the total space occupied by the muscle in relation to the total calf volume supporting our findings of total variance in $T_{2}$ of TA and soleus explained by ECF:ICF. Although ratio of ECF : ICF could significantly predict the $T_{2}$ of TA and soleus, the standard error of estimate was larger than the $\mathrm{MDC}_{95}$ value of $T_{2}$ of TA and soleus. Hence the predicted values for $T_{2}$ of TA and soleus using these equations may have larger error for estimating true value.

The $T_{2}$ of the muscles evaluated in this study ranged from $36.37 \mathrm{~ms}$ for TA to $40.13 \mathrm{~ms}$ for the soleus muscles. This reflects the differences in the fiber-type composition of these 
Difference versus average: apparent diffusion coefficient (ADC)

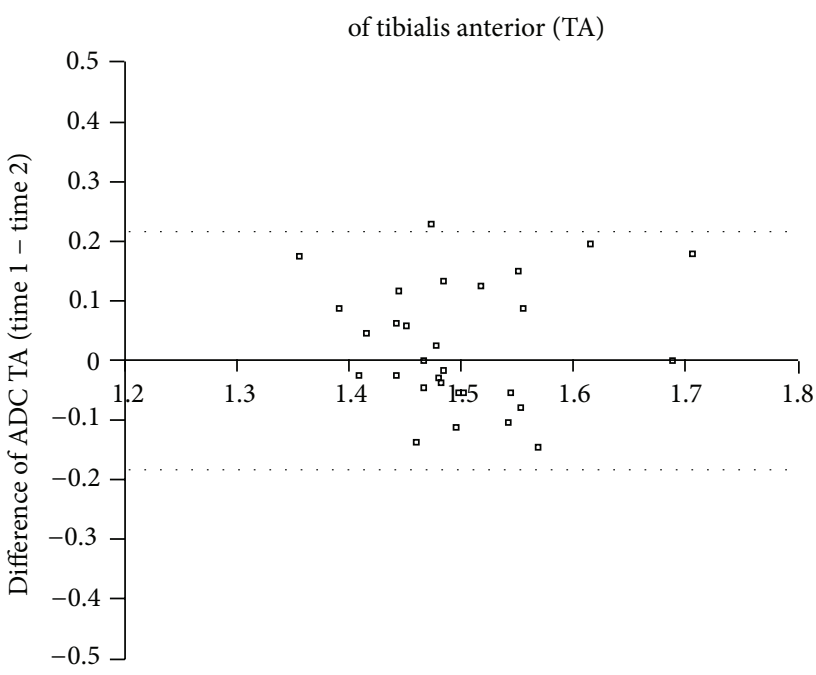

Average ADC TA (time $1+$ time 2)/2

Difference versus average: apparent diffusion coefficient (ADC) of medial gastrocnemius (MG)

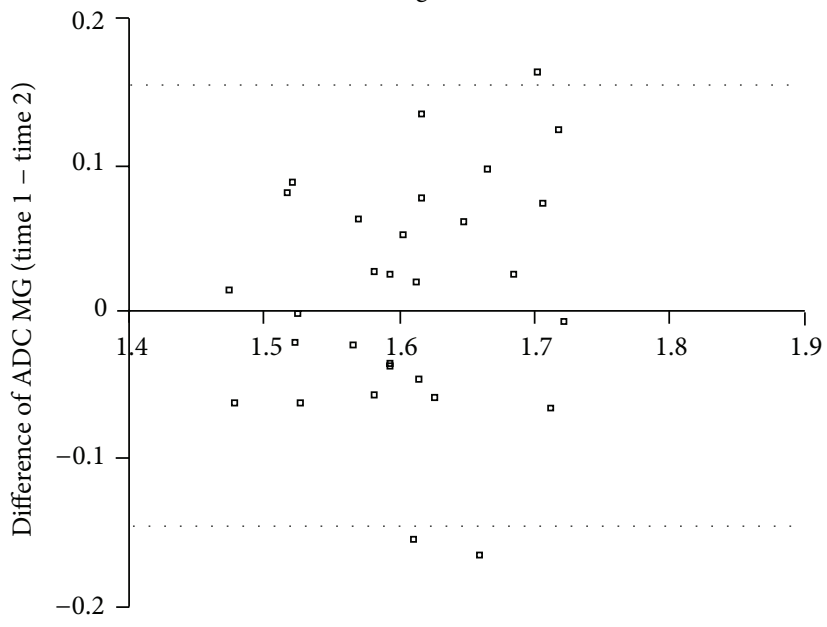

Average ADC MG (time $1+$ time 2)/2
Difference versus average: apparent diffusion coefficient (ADC)

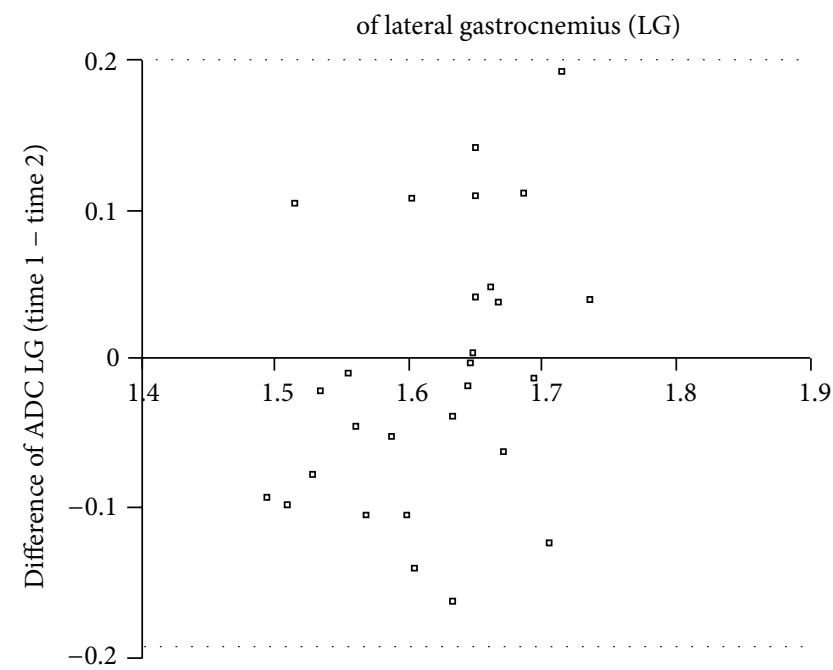

Average ADC LG (time $1+$ time 2)/2

Difference versus average: apparent diffusion coefficient (ADC) of soleus

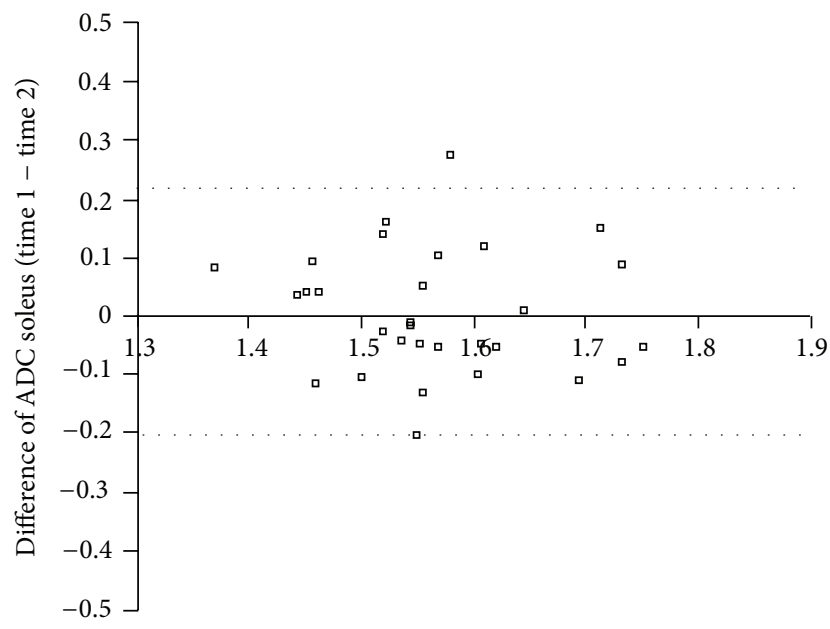

Average ADC soleus (time $1+$ time 2)/2

FIGURE 6: Plots of difference versus average: apparent diffusion coefficient measures (ADC).

muscles. The heterogeneous muscle fiber composition and plasticity of skeletal muscles (ability to change the fibre type composition in response to environment/physical activity) can affect estimates of $T_{2}$ as MRI signals are strongly related to the histochemical composition of the tissue [49]. These findings of heterogeneous $T_{2}$ are in accordance with priorpublished reports investigating $T_{2}$ of various muscles. For example subscapularis muscle $T_{2}(31.5 \mathrm{~ms})$ was slightly higher than anterior deltoid $(29.5 \mathrm{~ms})$; the same authors showed a change of $10 \mathrm{~ms}$ in the supraspinatus muscle $T_{2}$ following three sessions of exercise intervention (empty-can and fullcan exercise) performed within one week [50]. Additionally, the diffusion rate of muscle cell membrane that takes place in the presence of magnetic local field in-homogeneities can impact the $T_{2}[17]$.
The poor reliability of ADC measures can be supported by the fact that body fluids are rarely stable. Study of volume of body fluid compartments, ICF and ECF concentration, using isotope dilution methods, requires several hours to equilibrate (3-5 hrs) the isotopes [39]. For practical reasons we allowed only 30 minutes for body fluids to equilibrate prior to acquiring MRI data, and this may have perhaps affected the reliability of the ADC measures. However, as ADC can be influenced by changes in the osmolality, change in ADC may be more sensitive to the changes in microcirculation associated with activity or hydration status of the muscle, and a difference greater than 0.1 to $0.12 \mathrm{~mm}^{2} / \mathrm{s}$ would indicate a true change on repeated measurements [35]. Following eccentric exercise interventions, Yanagisawa et al. [22] were able to demonstrate significant change in ADC values of $\mathrm{LG}$ 
TABLE 7: Results of agreement between cBIS and MRI-acquired measures.

\begin{tabular}{lcccccccccc}
\hline & \multicolumn{2}{c}{$R$} & \multicolumn{2}{c}{$R^{2}$} & \multicolumn{2}{c}{ SEE } & \multicolumn{2}{c}{ Constant (95\% CI) } & \multicolumn{2}{c}{ cBIS ECF : ICF (95\% CI) } \\
& $T_{2}$ & ADC & $T_{2}$ & ADC & $T_{2}$ & ADC & $T_{2}$ & ADC & $T_{2}$ & ADC \\
\hline cBIS ECF : ICF & & & & & & & & & & \\
TA & $0.36^{*}$ & 0.12 & $0.13^{*}$ & 0.01 & 1.45 & 0.08 & $33.6^{*}(31.2,36.1)$ & $1.5(1.3,1.6)$ & $8.3^{*}(0.4,16.3)$ & $0.1(-0.3,0.6)$ \\
LG & 0.29 & 0.08 & 0.08 & 0.01 & 3.39 & 0.1 & $35.7(29.9,41.4)$ & $1.6(1.4,1.7)$ & $15.0(-3.4,33.5)$ & $0.1(-0.4,0.7)$ \\
MG & 0.28 & 0.15 & 0.08 & 0.02 & 3.8 & 0.07 & $33.9(27.6,40.4)$ & $1.6(1.4,1.7)$ & $17.2(-19.1,53.4)$ & $0.16(-0.2,0.6)$ \\
Soleus & $0.41^{*}$ & 0.22 & $0.17^{*}$ & 0.05 & 3.1 & 0.09 & $33.8(28.8,38.8)$ & $1.66(1.51,1.82)$ & $19.8^{*}(3.6,35.9)$ & $-0.3(-0.81,0.21)$ \\
\hline
\end{tabular}

${ }^{*} P<0.05$.

ADC: apparent diffusion coefficient; cBIS: calf bioelectrical impedance spectroscopy; CI: confidence interval; ECF: extracellular fluid; ICF: intracellular fluid; LG: lateral gastrocnemius; MG: medial gastrocnemius; $R$ : correlation coefficient; $R^{2}:$ coefficient of determination; $T_{2}$ : transverse relaxation time constants; SEE: standard error of estimate.
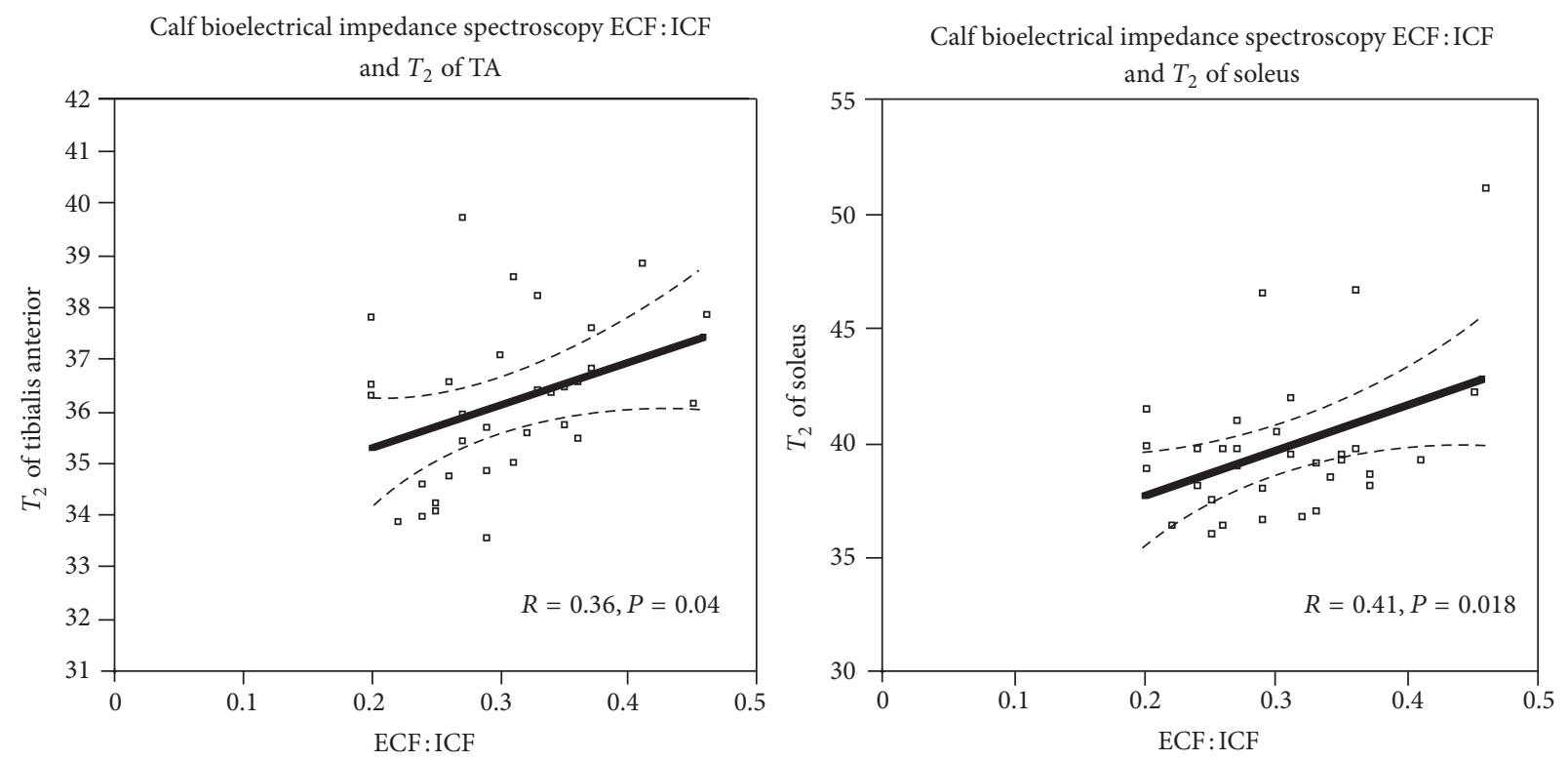

FIgURE 7: Graph of ECF : ICF by $T_{2}$ of tibialis anterior and soleus.

that reflected physiological changes affecting fluid movement associated with such exercises.

Our results can be applied to the studies utilizing similar methods of selecting smaller ROI and using OsiriX for data analysis with data collected as described in the methods section [51]. A review of literature indicates differences in the techniques of measurement of $T_{2}$. For example, Liu et al. [52] measured $T_{2}$ of the rat TA, gastrocnemius, and soleus using in-house software, and the whole muscle boundaries were outlined as regions of interest; Hatakenaka et al. [18] selected ROIs between 20 and $30 \mathrm{~mm}^{2}$ for each muscle and calculated $T_{2}$ by fitting the appropriate signal intensities to an equation. Hence, it would be inappropriate to compare our findings with results of their studies.

\section{Conclusions}

The findings of our study indicate that the MRI-acquired $T_{2}$ of the muscles of the lower leg has excellent test-retest reliability and is appropriate for studies utilizing repeated measurements. The relative variability of this measure is smaller than the cBIS-acquired measures and hence can be utilized in studies recruiting participants of varying age. These results can be applied to studies utilizing similar techniques for data collection and analysis. The ADC of the leg muscles can be used in studies with relatively large sample sizes, as ADC may be sensitive to minor variation in the circulation of the muscles associated with variations in body fluids and activity. Although cBIS-acquired measures have excellent test-retest reliability, whether these measures can be used to estimate interstitial fluid of a single muscle requires further investigations.

\section{Disclosures}

Anuradha Sawant was a recipient of the Allied Health Doctoral Fellowship from The Kidney Foundation of Canada.

\section{Conflict of Interests}

The authors declare that they have no conflict of interests. 


\section{Acknowledgments}

Thanks are due to the Kidney Foundation of Canada for providing funding for this study, The Renal Research Institute of New York for providing training, software, and bioelectrical impedance spectroscopy equipment to measure calf hydration, Kim Krueger and Oksana Opaleych for their patience while the author worked on refining the protocol and all the participants for their time and support for this study.

\section{References}

[1] Q.-L. Zhang and D. Rothenbacher, "Prevalence of chronic kidney disease in population-based studies: systematic review," BMC Public Health, vol. 8, no. article 117, 2008.

[2] D. S. C. Raj, Y. Sun, and A. H. Tzamaloukas, "Hypercatabolism in dialysis patients," Current Opinion in Nephrology and Hypertension, vol. 17, no. 6, pp. 589-594, 2008.

[3] K. L. Johansen, T. Shubert, J. Doyle, B. Soher, G. K. Sakkas, and J. A. Kent-Braun, "Muscle atrophy in patients receiving hemodialysis: effects on muscle strength, muscle quality, and physical function," Kidney International, vol. 63, no. 1, pp. 291-297, 2003.

[4] K. L. Johansen, "Exercise in the end-stage renal disease population," Journal of the American Society of Nephrology, vol. 18, no. 6, pp. 1845-1854, 2007.

[5] N. Smart and M. Steele, "Exercise training in haemodialysis patients: a systematic review and meta-analysis," Nephrology, vol. 16, no. 7, pp. 626-632, 2011.

[6] A. Sawant, A. A. House, and T. J. Overend, "Anabolic effect of exercisetraining in people with end-stage renal disease on haemodialysis: systematic review and meta-analysis," Physiotherapy Canada. In press.

[7] R. F. Kushner, P. M. J. M. de Vries, and R. Gudivaka, "Use of bioelectrical impedance analysis measurements in the clinical management of patients undergoing dialysis," American Journal of Clinical Nutrition, vol. 64, no. 3, pp. 503s-509s, 1996.

[8] G. A. Kaysen, F. Zhu, S. Sarkar et al., "Estimation of total-body and limb muscle mass in hemodialysis patients by using multifrequency bioimpedance spectroscopy," American Journal of Clinical Nutrition, vol. 82, no. 5, pp. 988-995, 2005.

[9] B. Charra, "Fluid balance, dry weight, and blood pressure in dialysis," Hemodialysis International, vol. 11, no. 1, pp. 21-31, 2007.

[10] E. Lindley, L. Aspinall, and C. Gardiner E Garthwaite, "Management of fluid status in haemodialysis patients: the roles of technology and dietary advice," in Technical Problems in Patients on Hemodialysis, M. G. Penido, Ed., InTech, Rijeka, Croatia, 2011.

[11] DaVita, “The hemodialysis diet," http://www.davita.com/ kidney-disease/diet-andnutrition/diet-basics/the-hemodialysis-diet/e/5314.

[12] P. MacHek, T. Jirka, U. Moissl, P. Chamney, and P. Wabel, "Guided optimization of fluid status in haemodialysis patients," Nephrology Dialysis Transplantation, vol. 25, no. 2, pp. 538-544, 2010.

[13] J. Passauer, E. Bussemaker, and P. Gross, "Dialysis hypotension: do we see light at the end of the tunnel?" Nephrology Dialysis Transplantation, vol. 13, no. 12, pp. 3024-3029, 1998.

[14] A. K. Jain and R. M. Lindsay, "Intra and extra cellular fluid shifts during the inter dialytic period in conventional and daily hemodialysis patients," ASAIO Journal, vol. 54, no. 1, pp. 100 103, 2008.

[15] D. A. Judelson, C. M. Maresh, J. M. Anderson et al., "Hydration and muscular performance: does fluid balance affect strength, power and high-intensity endurance?" Sports Medicine, vol. 37, no. 10, pp. 907-921, 2007.

[16] P. Kotanko, N. W. Levin, and F. Zhu, "Current state of bioimpedance technologies in dialysis," Nephrology Dialysis Transplantation, vol. 23, no. 3, pp. 808-812, 2008.

[17] C. Patten, R. A. Meyer, and J. L. Fleckenstein, “ $\mathrm{T}_{2}$ Mapping of Muscle," Seminars in Musculoskeletal Radiology, vol. 7, no. 4, pp. 297-305, 2003.

[18] M. Hatakenaka, H. Soeda, T. Okafuji et al., "Steroid myopathy: evaluation of fiber atrophy with $\mathrm{T}_{2}$ relaxation time-rabbit and human study," Radiology, vol. 238, no. 2, pp. 650-657, 2006.

[19] N. Holl, A. Echaniz-Laguna, G. Bierry et al., "Diffusion-weighted MRI of denervated muscle: a clinical and experimental study," Skeletal Radiology, vol. 37, no. 12, pp. 1111-1117, 2008.

[20] J. F. Polak, F. A. Jolesz, and D. F. Adams, "Magnetic resonance imaging of skeletal muscle. Prolongation of $\mathrm{T}_{1}$ and $\mathrm{T}_{2}$ subsequent to denervation," Investigative Radiology, vol. 23, no. 5, pp. 365-369, 1988.

[21] A. T. Nygren and L. Kaijser, "Water exchange induced by unilateral exercise in active and inactive skeletal muscles," Journal of Applied Physiology, vol. 93, no. 5, pp. 1716-1722, 2002.

[22] O. Yanagisawa, D. Shimao, K. Maruyama, M. Nielsen, T. Irie, and M. Niitsu, "Diffusion-weighted magnetic resonance imaging of human skeletal muscles: gender-, age- and musclerelated differences in apparent diffusion coefficient," Magnetic Resonance Imaging, vol. 27, no. 1, pp. 69-78, 2009.

[23] U. G. Kyle, I. Bosaeusb, and A. D. de Lorenzoc, "Bioelectrical impedance analysis part I: review of principles and methods," Clinical Nutrition, vol. 23, no. 5, pp. 1226-1243, 2004.

[24] J. M. Bland and D. G. Altman, "Measuring agreement in method comparison studies," Statistical Methods in Medical Research, vol. 8, no. 2, pp. 135-160, 1999.

[25] A. Donner and M. Eliasziw, "Sample size requirements for reliability studies," Statistics in Medicine, vol. 6, no. 4, pp. 441448, 1987.

[26] A. Fix and D. Daughton, Human Activity Profile, Psychological Assessment Resources, 1988.

[27] W. H. Hall, R. Ramachandran, S. Narayan, A. B. Jani, and S. Vijayakumar, "An electronic application for rapidly calculating Charlson comorbidity score," BMC Cancer, vol. 4, article 94, 2004.

[28] H. Gray, "Anatomy of the human body," http://www.bartleby .com/107/129.html.

[29] F. Zhu, M. K. Kuhlmann, S. Sarkar et al., "Adjustment of dry weight in hemodialysis patients using intradialytic continuous multifrequency bioimpedance of the calf," International Journal of Artificial Organs, vol. 27, no. 2, pp. 104-109, 2004.

[30] E. le Rumeur, F. Carre, A.-M. Bernard, J.-Y. Bansard, P. Rochcongar, and J. D. de Certaines, "Multiparametric classification of muscle $\mathrm{T}_{1}$ and $\mathrm{T}_{2}$ relaxation times determined by magnetic resonance imaging. The effects of dynamic exercise in trained and untrained subjects," British Journal of Radiology, vol. 67, no. 794, pp. 150-156, 1994.

[31] H. E. Berg, B. Tedner, and P. A. Tesch, "Changes in lower limb muscle cross-sectional area and tissue fluid volume after transition from standing to supine," Acta Physiologica Scandinavica, vol. 148, no. 4, pp. 379-385, 1993. 
[32] P. E. Shrout and J. L. Fleiss, "Intraclass correlations: uses in assessing rater reliability," Psychological Bulletin, vol. 86, no. 2, pp. 420-428, 1979.

[33] D. L. Streiner and G. R. Norman, "Reliability," in Health Measurement Scales: Practical Guide to Their Development and Use, pp. 126-152, Oxford University Press, Oxford, UK, 3rd edition, 2003.

[34] P. W. Stratford and D. L. Riddle, "When minimal detectable change exceeds a diagnostic test-based threshold change value for an outcome measure: resolving the conflict," Physical Therapy, vol. 92, no. 10, pp. 1338-1347, 2012.

[35] P. W. Stratford and C. H. Goldsmith, "Use of the standard error as a reliability index of interest: an applied example using elbow flexor strength data," Physical Therapy, vol. 77, no. 7, pp. 745-750, 1997.

[36] D. Cramer, "Levene's test," in The Sage Encyclopedia of Social Science Research Methods, M. S. L. Beck, A. Bryman, and T. F. Lio, Eds., 2004.

[37] J. M. Bland and D. G. Altman, "Applying the right statistics: analyses of measurement studies," Ultrasound in Obstetrics and Gynecology, vol. 22, no. 1, pp. 85-93, 2003.

[38] M. Dittmar, "Reliability and variability of bioimpedance measures in normal adults: effects of age, gender, and body mass," American Journal of Physical Anthropology, vol. 122, no. 4, pp. 361-370, 2003.

[39] L. E. Armstrong, "Assessing hydration status: the elusive gold standard," Journal of the American College of Nutrition, vol. 26, no. 5, pp. 575S-584S, 2007.

[40] Y. Yamada, D. A. Schoeller, E. Nakamura, T. Morimoto, M. Kimura, and S. Oda, "Extracellular water may mask actual muscle atrophy during aging," Journals of Gerontology A, vol. 65, no. 5, pp. 510-516, 2010.

[41] I. R. Lanza, D. W. Russ, and J. A. Kent-Braun, "Age-related enhancement of fatigue resistance is evident in men during both isometric and dynamic tasks," Journal of Applied Physiology, vol. 97, no. 3, pp. 967-975, 2004.

[42] C. J. McNeil, T. J. Doherty, D. W. Stashuk, and C. L. Rice, "Motor unit number estimates in the tibialis anterior muscle of young, old, and very old men," Muscle and Nerve, vol. 31, no. 4, pp. 461467, 2005.

[43] C. J. McNeil and C. L. Rice, "Fatigability is increased with age during velocity-dependent contractions of the dorsiflexors," Journals of Gerontology A, vol. 62, no. 6, pp. 624-629, 2007.

[44] A. A. Vandervoort and A. J. McComas, "Contractile changes in opposing muscles of the human ankle joint with aging," Journal of Applied Physiology, vol. 61, no. 1, pp. 361-367, 1986.

[45] G. B. Joseph, T. Baum, J. Carballido-Gamio et al., "Texture analysis of cartilage $T_{2}$ maps: individuals with risk factors for OA have higher and more heterogeneous knee cartilage MR $\mathrm{T}_{2}$ compared to normal controls-data from the osteoarthritis initiative," Arthritis Research \& Therapy, vol. 13, article R153, 2011.

[46] S. B. Heymsfield, D. Gallagher, M. Visser, C. Nunez, and Z.M. Wang, "Measurement of skeletal muscle: laboratory and epidemiological methods," Journals of Gerontology A, vol. 50, pp. 23-29, 1995.

[47] P. A. Baulby and F. J. Rugg-Gunn, “ $\mathrm{T}_{2}$ : the transverse relaxation time," in Quantitative MRI of the Brain Measuring Changes Caused By Disease, P. Tofts, Ed., pp. 143-201, John Wiley and Sons, Chinchester, UK, 2004.

[48] K. Brandis, "Fluid physiology", http://www.anaesthesiamcq .com/FluidBook/fl2_1.php.
[49] R. L. Segal, "Use of imaging to assess normal and adaptive muscle function," Physical Therapy, vol. 87, no. 6, pp. 704-718, 2007.

[50] Y. Takeda, S. Kashiwaguchi, K. Endo, T. Matsuura, and T. Sasa, "The most effective exercise for strengthening the supraspinatus muscle. Evaluation by magnetic resonance imaging," American Journal of Sports Medicine, vol. 30, no. 3, pp. 374-381, 2002.

[51] C. S. Poon and R. M. Henkelman, "Practical $T_{2}$ quantitation for clinical applications," Journal of Magnetic Resonance Imaging, vol. 2, no. 5, pp. 541-553, 1992.

[52] M. Liu, P. Bose, G. A. Walter, D. K. Anderson, F. J. Thompson, and $\mathrm{K}$. Vandenborne, "Changes in muscle $\mathrm{T}_{2}$ relaxation properties following spinal cord injury and locomotor training," European Journal of Applied Physiology, vol. 97, no. 3, pp. 355-361, 2006. 

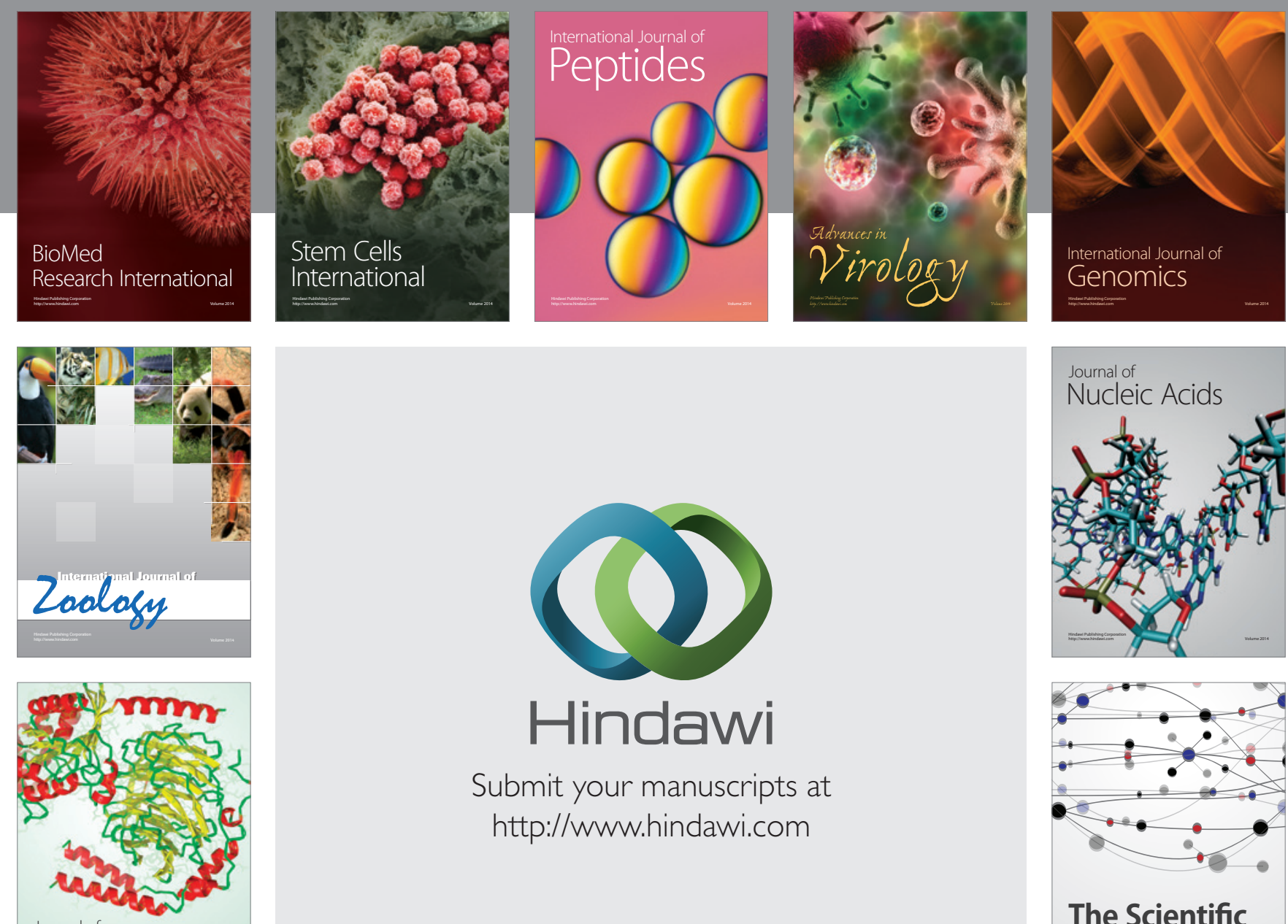

Submit your manuscripts at

http://www.hindawi.com

Journal of
Signal Transduction
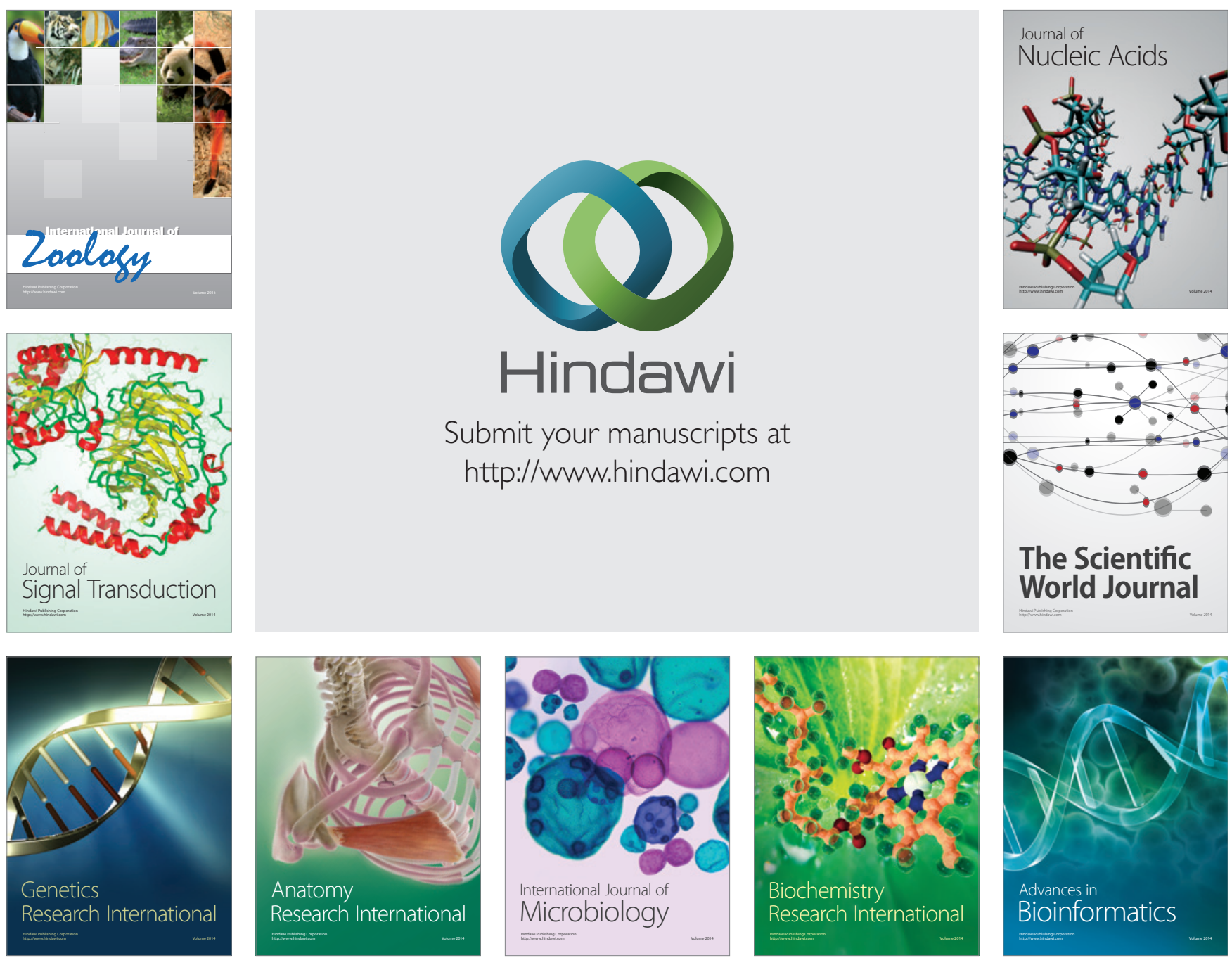

The Scientific World Journal
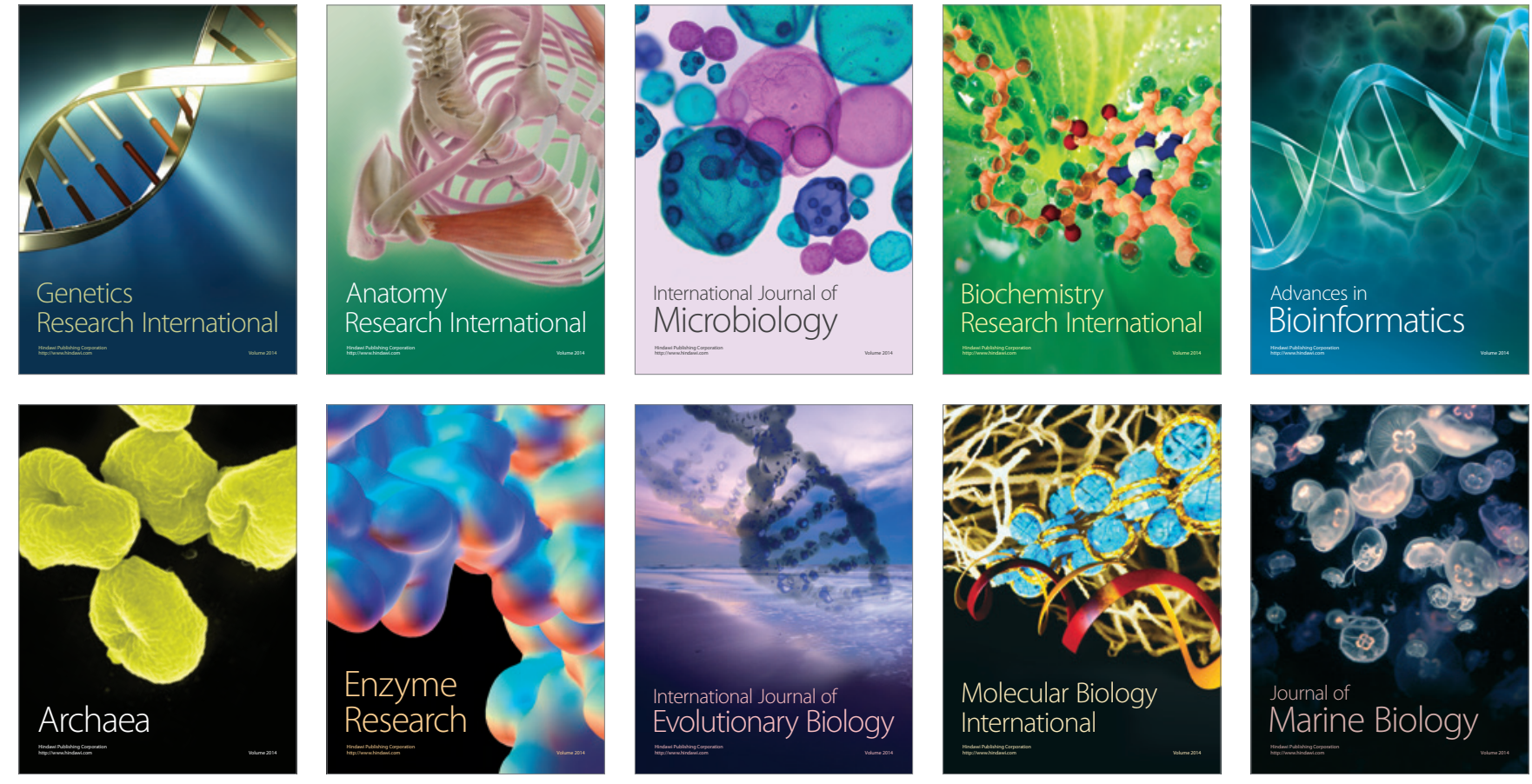